The Astrophysical Journal, 633:358-366, 2005 November 1

(C) 2005. The American Astronomical Society. All rights reserved. Printed in U.S.A.

\title{
THE ISLAND STATE OF THE ATOLL SOURCE 4U 1820-30
}

\author{
Diego Altamirano, ${ }^{1}$ M. van der Klis, ${ }^{1}$ M. Méndez, ${ }^{2}$ S. Migliari, ${ }^{1}$ P. G. Jonker, ${ }^{2,3}$ A. Tiengo, ${ }^{4,5}$ and W. Zhang ${ }^{6}$ \\ Received 2005 April 12; accepted 2005 July 11
}

\begin{abstract}
We study the rapid X-ray time variability in all public data available from the Rossi X-Ray Timing Explorer Proportional Counter Array on the atoll source 4U 1820-30 in the low-luminosity island state. A total of $\sim 46 \mathrm{ks}$ of data were used. We compare the frequencies of the variability components of $4 \mathrm{U} 1820-30$ with those in other atoll sources. These frequencies were previously found to follow a universal scheme of correlations. We find that $4 \mathrm{U}$ 1820-30 shows correlations that are shifted by factors of $1.13 \pm 0.01$ and $1.21 \pm 0.02$ with respect to those in other atoll sources. These shifts are similar to, but smaller than, the shift factor of $\sim 1.45$ previously reported for some accreting millisecond pulsars. Therefore, $4 \mathrm{U} 1820-30$ is the first atoll source that shows no significant pulsations but has a significant shift in the frequency correlations compared with the other three nonpulsating atoll sources.
\end{abstract}

Subject headings: accretion, accretion disks — binaries: close — stars: individual (4U 0614+09, 4U 1608-52, 4U 1728-34, 4U 1820-30, SAX J1808.4-3658) — stars: neutron — X-rays: stars

\section{INTRODUCTION}

Accretion in neutron star low-mass X-ray binaries (LMXBs) can be studied through the spectral and timing properties of the associated X-ray emission. The Fourier power spectra of the $\mathrm{X}$-ray flux of these systems exhibit quasi-periodic oscillations (QPOs) as well as noise components between $\sim 1 \times 10^{-3}$ and $\sim 1350 \mathrm{~Hz}$. Most of these variability components are thought to be associated with processes in the accretion disk (for reviews and references, see van der Klis 2000, 2006), but some of them may arise on the surface of the neutron star (see, e.g., Revnivtsev et al. 2001; Strohmayer \& Bildsten 2006). The timing properties at low frequencies $(\nu<100 \mathrm{~Hz})$ as well as the spectral properties are the basis of the classification of these systems as either $\mathrm{Z}$ or atoll sources (Hasinger \& van der Klis 1989). In recent literature, each variability component is designated $L_{i}$ ( $L$ for Lorentzian; Belloni et al. 2002), where the index $i$ indicates the component; the component's characteristic frequency is designated $\nu_{i}$. For example, $L_{b}$ is an often flat-topped broadband noise component at a low frequency $\nu_{b}$, and $L_{u}$ is the upper kilohertz QPO with frequency $\nu_{u}$. (See van Straaten et al. [2003] for complete terminology.)

The kilohertz QPOs are seen between a few hundred and $\sim 1350 \mathrm{~Hz}$, and when two of them are seen at the same time (twin kilohertz QPOs), the difference between their frequencies is constrained between $\sim 185$ and $\sim 400 \mathrm{~Hz}$. In the $0.01-100 \mathrm{~Hz}$ range, two to five band-limited noise, peaked-noise, and QPO components are observed whose frequencies all correlate with one another and with that of the kilohertz QPOs (see van

\footnotetext{
1 Astronomical Institute Anton Pannekoek, University of Amsterdam and Center for High-Energy Astrophysics, Kruislaan 403, NL 1098 SJ Amsterdam, Netherlands; diego@science.uva.nl.

2 SRON National Institute for Space Research, Sorbonnelaan 2, 3584 CA Utrecht, Netherlands.

3 Harvard-Smithsonian Center for Astrophysics, 60 Garden Street, MS 83, Cambridge, MA 02138.

4 INAF-Istituto di Astrofisica Spaziale e Fisica Cosmica, Sezione di Milano “G.Occhialini," Via Bassini 15, I-20133 Milan, Italy.

5 Università degli Studi di Milano, Dipartimento di Fisica, Via Celoria 16, I-20133 Milan, Italy.

6 Laboratory for X-Ray Astrophysics, NASA Goddard Space Flight Center, Greenbelt, MD 20771.
}

Straaten et al. 2005 and references therein). An example is the WK correlation (after Wijnands \& van der Klis 1999) between the hump frequency $\nu_{h}$ and the break frequency $\nu_{b}$. This relation may be fundamental in understanding the processes of accretion in LMXBs, because in atoll sources and black holes $\nu_{b}$ and $\nu_{h}$ correlate over 3 orders of magnitude (the $\mathrm{Z}$ sources have slightly higher $\nu_{h}$ ). The existence of such correlations suggests that similar physical phenomena may be responsible for some of the QPOs and noise components found over wide ranges of frequency and coherence in Z, atoll, and black hole sources.

Authors van Straaten et al. (2005) found that the frequencies of the noise and QPO components of the accreting millisecond pulsar SAX J1808.4-3658 also correlate with $\nu_{u}$ but in a different way from those of the other atoll sources. They interpreted the difference between the pulsar and the atoll sources as being due to a shift in frequency of the upper kilohertz QPO and suggested that physical differences between these sources are most likely to affect the high-frequency components. In SAX J1808.4-3658, the factors by which $\nu_{u}$ had to be multiplied to make the correlations coincide with those of the ordinary atoll sources were $1.420 \pm 0.013$ for $\nu_{b}$ and $1.481 \pm 0.013$ for $\nu_{h}$.

$4 \mathrm{U} 1820-30$ is a LMXB with an orbital period of only 11.4 minutes (Stella et al. 1987) and an X-ray burst source (Grindlay et al. 1976). It is located in the globular cluster NGC 6624 at a distance of $7.6 \pm 0.4 \mathrm{kpc}$ (Kuulkers et al. 2003). Radio emission has also been detected from the source (Geldzahler 1983; Migliari et al. 2004). 4U 1820-30 undergoes a regular $\sim 176$ day accretion cycle (Priedhorsky \& Terrell 1984), switching between high and low states differing by a factor of $\sim 3$ in luminosity (Strohmayer \& Brown 2002). The ultracompact nature of the system requires that the secondary is a low-mass helium dwarf (see, e.g., Rappaport et al. 1987), so that the accreted material likely has a very low hydrogen abundance. Hasinger \& van der Klis (1989) defined 4U 1820-30 as an atoll source, and 8 years later Smale et al. (1997) reported the discovery of kilohertz quasi-periodic oscillations. Zhang et al. (1998) reported the result from a long-term monitoring data set obtained with the Rossi X-Ray Timing Explorer (RXTE). They observed kilohertz QPOs in both the lower banana and the island state (see van der Klis [2006] for nomenclature). They showed that the frequency of the kilohertz QPOs is correlated with the 
Proportional Counter Array (PCA) count rate below a critical value ( $\sim 2500$ counts $\mathrm{s}^{-1}$ per 5 Proportional Counter Units [PCUs]). Above this, the QPO frequencies remained constant while the count rate increased between $\sim 2500$ and $\sim 3200$ counts s$^{-1}$ per 5 PCUs. Saturation of QPO frequency at high mass accretion rates is an expected signature of the marginally stable orbit (Miller et al. 1998; Kaaret et al. 1999); however, this is the only source reported to have shown this behavior, and to what extent count rate is a good indicator of accretion rate remains to be seen (see, e.g., the discussion of the issue in van der Klis 2001). Later, similar analyses were carried out, using instead of the count rate (1) the energy flux, (2) the X-ray spectral shape (Kaaret et al. 1999), and (3) the parameter $S_{a}$ (Bloser et al. 2000), which parameterizes atoll source location in the track of the color-color diagram. The same behavior as that observed by Zhang et al. (1998) as a function of count rate was found when the QPO frequency was plotted as a function of either of these three parameters. The saturation of QPO frequency was interpreted as strong additional evidence for the detection of the marginally stable orbit in the accretion disk of $4 \mathrm{U} 1820-30$. However, since then Méndez (2002) has argued that the evidence of the saturation is not so compelling, especially when some instrumental corrections are taken into account. A general tendency for QPO frequency to saturate toward higher luminosity may be a feature of the same phenomenon that produces the parallel tracks in frequency-luminosity diagrams (van der Klis 2001).

In this paper, we report on the eight observations that are currently available of $4 \mathrm{U} 1820-30$ in the island state. All previous works mentioned above included only one observation (20075-01-05-00) of the source in the island state, so the current analysis better allows us to constrain the power spectral components in the island state of $4 \mathrm{U} 1820-30$ more accurately. We study the correlations between the characteristic frequencies of the various timing features and compare these with those of four well-studied atoll sources, three low-luminosity bursters, one $\mathrm{Z}$ source, and one accreting millisecond pulsar. We show that the correlations between frequencies in $4 \mathrm{U} 1820-30$ are shifted as found for SAX J1808.4-3658, but with a lower shift factor. We finally discuss whether the interpretation of a multiplicative shift of frequencies is the right explanation for the differences in frequency behavior between the millisecond accreting pulsar SAX J1808.4-3658 and the ordinary atoll sources.

\section{OBSERVATIONS AND DATA ANALYSIS}

We used all public data available from the RXTE PCA (for instrument information, see Zhang et al. 1993). There were 158 pointed observations in 11 programs $(10074,10075,10076$, $20075,30053,30057,40017,40019,60030,70030$, and 70031). In our analysis, we use the $16 \mathrm{~s}$ time resolution Standard 2 mode data to calculate X-ray colors. For each of the five PCA detectors (PCUs) we calculate a hard and a soft color, defined as the count rate in the $9.7-16.0 \mathrm{keV}$ band divided by the rate in the $6.0-9.7 \mathrm{keV}$ band, and the $3.5-6.0 \mathrm{keV}$ rate divided by the $2.0-$ $3.5 \mathrm{keV}$ rate, respectively. For each detector we also calculate the intensity, defined as the count rate in the energy band 2.0$16 \mathrm{keV}$. To obtain the count rates in these exact energy ranges, we interpolate linearly between count rates in the PCU channels. We then subtract the background contribution in each band using the standard bright source background model for the PCA, version 2.1 e. ${ }^{7}$ No dead-time corrections were made, as the effect of dead time can be neglected for our purposes $(<0.001 \%)$. We calculate

\footnotetext{
${ }^{7}$ For details of the model, see the PCA Digest at http://heasarc.gsfc.nasa
}

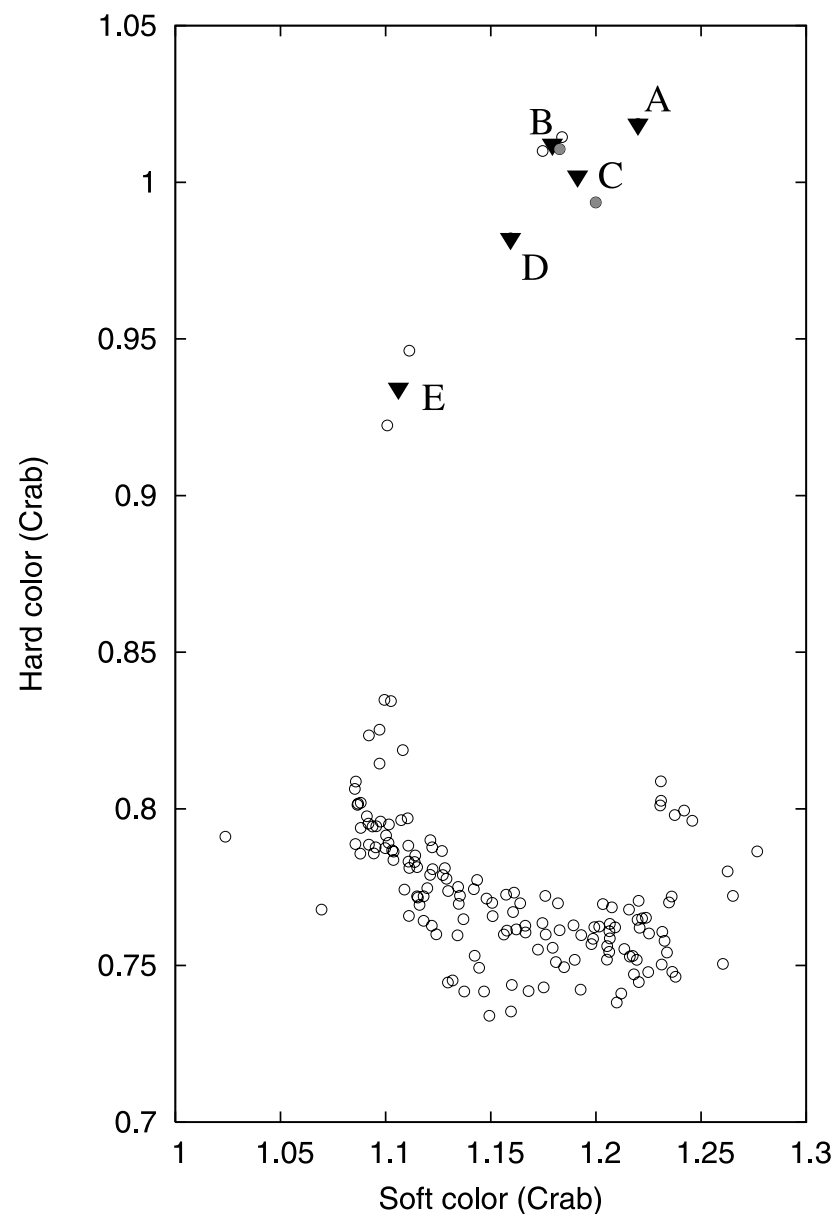

FIG. 1. - $4 \mathrm{U} 1820-30$ 's hard color vs. soft color normalized to Crab colors as explained in $\S 1$. Each circle represents one of the 158 observations mentioned in $\S 2$. The triangles represent the average power spectra A-E. They correspond to one or two observations and are labeled in order of decreasing average hard color. For clarity, the two gray circles represent the two observations averaged to get power spectrum C. The error bars are of the order of the size of the symbols.

the colors and intensity for each time interval of $16 \mathrm{~s}$. In order to correct for the gain changes as well as the differences in effective area between the PCUs themselves, we used the method introduced by Kuulkers et al. (1994): for each PCU we calculate, in the same manner as for $4 \mathrm{U} 1820-30$, the colors of the Crab that can be supposed to be constant. We then average the $16 \mathrm{~s}$ Crab colors and intensity for each PCU for each day. For each PCU we divide the $16 \mathrm{~s}$ color and intensity values obtained for $4 \mathrm{U}$ $1820-30$ by the corresponding Crab values that are closest in time but in the same RXTE gain epoch. The RXTE gain epoch changes with each new high-voltage setting of the PCUs (Jahoda et al. 1996). After the Crab normalization is done, we average the colors and intensity over all PCUs. Finally, we average the $16 \mathrm{~s}$ colors per observation. Figure 1 shows the color-color diagram of the 158 different observations that we used for this analysis, and Figure 2 shows the corresponding hardness-intensity diagrams (soft and hard color vs. intensity). We find eight observations that are located in the island region of the color-color diagram (hard colors greater than 0.9). These observations are the subject of this paper (see Table 1).

For the Fourier timing analysis of these eight observations we used an $125 \mu$ s time resolution event mode (E_125us 64M_0_1s). Leahy-normalized power spectra were constructed using data segments of $128 \mathrm{~s}$ and $1 / 8192 \mathrm{~s}$ time bins such that 

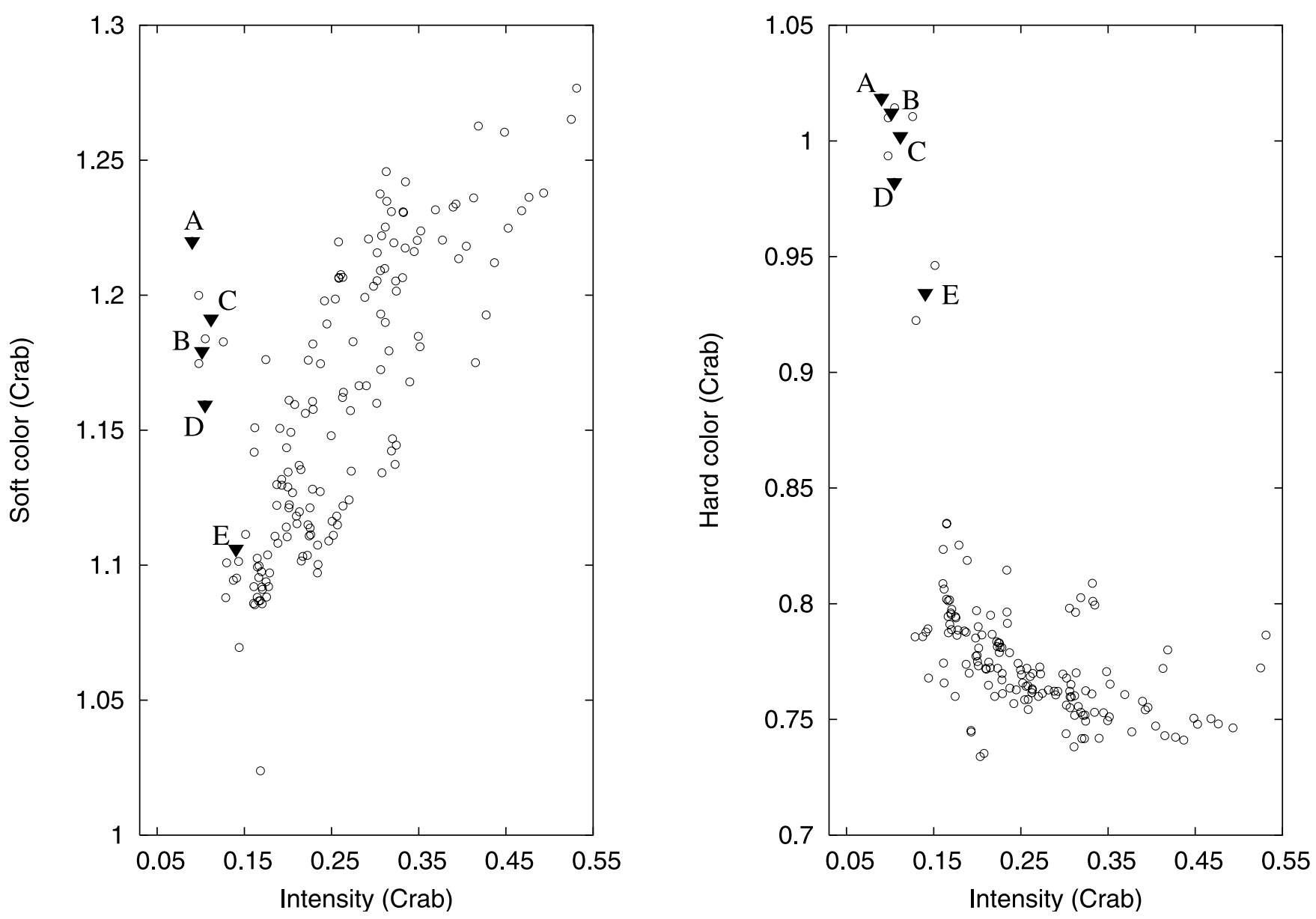

Fig. 2.-Soft color vs. intensity (left) and hard color vs. intensity (right) in Crab units as explained in $\S 1$. Symbols are the same as in Fig. 1. The error bars are of the order of the size of the symbols.

the lowest available frequency is $1 / 128 \approx 8 \times 10^{-3} \mathrm{~Hz}$ and the Nyquist frequency is $4096 \mathrm{~Hz}$. Detector dropouts were removed, but no background or dead-time corrections were performed prior to the calculation of the power spectra. We first averaged the power spectra per observation. We inspected the shape of the average power spectra at high frequency $(>2000 \mathrm{~Hz})$ for unusual features in addition to the usual Poisson noise. None were found. We then subtracted a Poisson noise spectrum estimated from the power between 3000 and $4000 \mathrm{~Hz}$, where neither intrinsic noise nor QPOs are known to be present, using the method developed by M. Klein-Wolt et al. (2005, in preparation) based on the analyti- cal function of Zhang et al. (1995). The resulting power spectra were then converted to squared fractional rms (van der Klis 1995). In this normalization the square root of the integrated power density is a direct measurement of the variance caused by the intrinsic variability in the source count rate. In three cases it was possible to add two observations together to improve statistics. This was done only for those observations that had similar colors and power spectra consistent with being the same within errors. The resulting power spectra are labeled from A to E (Fig. 3) in order of decreasing hard color. Table 1 shows which observations were used to create each of the averaged power spectra.

TABLE 1

The Eight Observations Used for the Timing Analysis

\begin{tabular}{|c|c|c|c|c|c|c|}
\hline Hard Color & Observation ID & Label & Date of Observation & $\begin{array}{l}\text { Duration } \\
(\mathrm{ks})\end{array}$ & $\begin{array}{l}\text { Total Average Count Rate } \\
\left(\text { counts } \mathrm{s}^{-1}\right)\end{array}$ & $\begin{array}{l}\text { Average Count Rate } \\
\text { (counts s }{ }^{-1} \mathrm{PCU}^{-1} \text { ) }\end{array}$ \\
\hline $1.018 \ldots \ldots \ldots \ldots \ldots \ldots \ldots \ldots$. & $40017-01-24-00$ & A & 2003 Jun 4 & $\sim 8.2$ & $\sim 720$ & $\sim 268$ \\
\hline 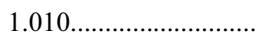 & 70030-03-04-00 & B & 2003 Jun 11 & $\sim 3.2$ & $\sim 1125$ & $\sim 281$ \\
\hline 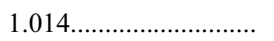 & $70030-03-05-00$ & B & 2003 Jun 14 & $\sim 6.5$ & $\sim 925$ & $\sim 308$ \\
\hline 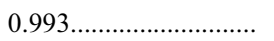 & 70030-03-04-01 & $\mathrm{C}$ & 2003 Jun 12 & $\sim 6.5$ & $\sim 988$ & $\sim 283$ \\
\hline 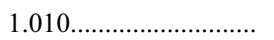 & $70030-03-05-01$ & $\mathrm{C}$ & 2003 Jun 15 & $\sim 6.6$ & $\sim 1076$ & $\sim 358$ \\
\hline 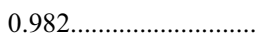 & 70031-05-01-00 & $\mathrm{D}$ & 2002 Jun 14 & $\sim 3.1$ & $\sim 1486$ & $\sim 297$ \\
\hline $0.922 \ldots \ldots \ldots \ldots \ldots \ldots \ldots \ldots$ & $20075-01-05-00$ & E & 1997 May 2 & $\sim 8.5$ & $\sim 1770$ & $\sim 354$ \\
\hline $0.946 \ldots \ldots \ldots \ldots \ldots \ldots \ldots$ & $70030-03-05-02$ & $\mathrm{E}$ & 2003 Jun 16 & $\sim 3.2$ & $\sim 1260$ & $\sim 421$ \\
\hline
\end{tabular}

NoтE.-The statistical errors in hard color are $\lesssim 0.001$. 

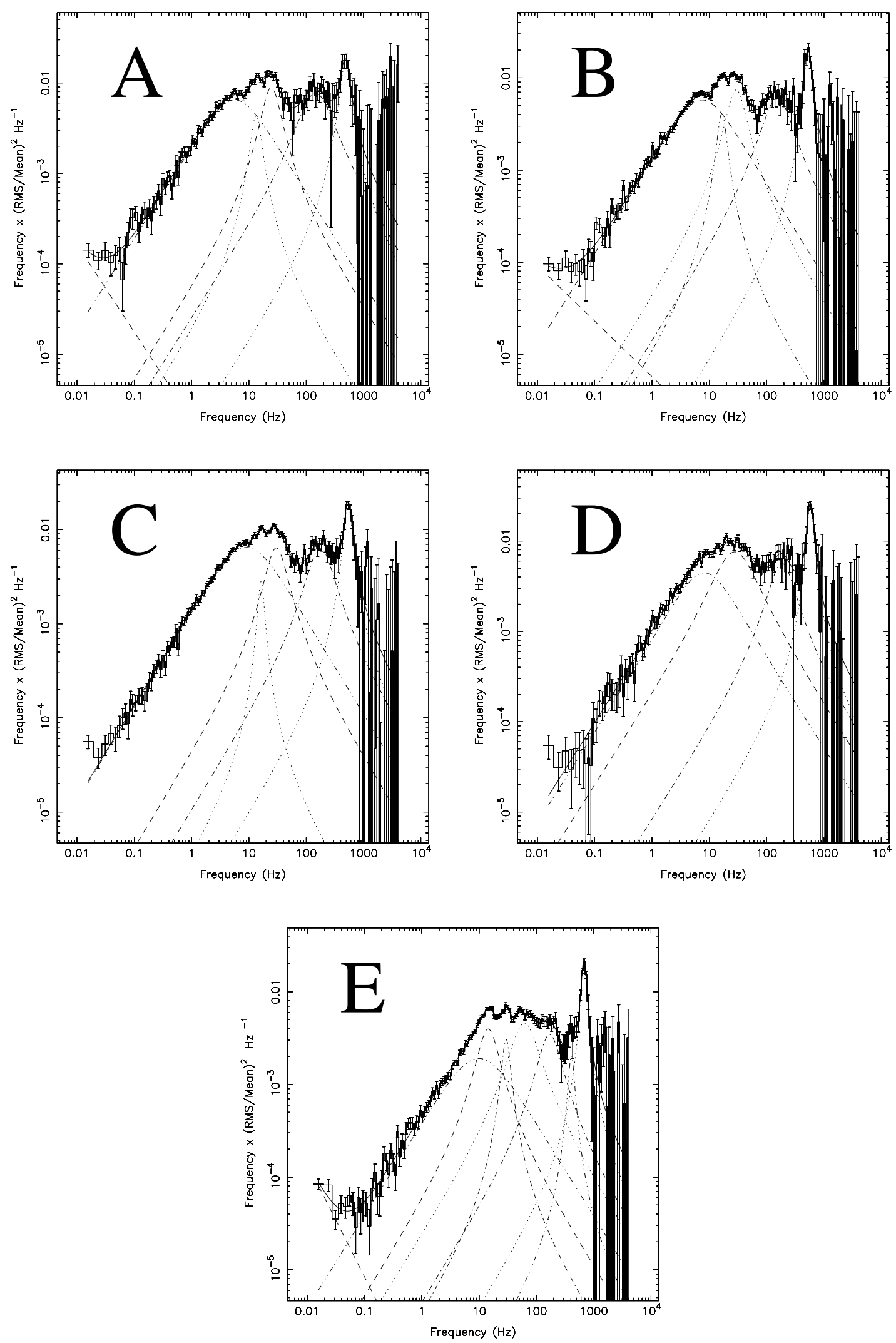

FIG. 3.-Power spectra and fit functions in the power spectral density times frequency representation for 4 U $1820-30$. Each plot corresponds to a different position in the color-color and color-intensity diagrams (see Figs. 1 and 2). The different lines mark the individual Lorentzian components of the fit. For a detailed identification, see Tables 2 and 3, Fig. 4, and $\S 3$. 
TABLE 2

Results of the Fits to the Power Spectra

\begin{tabular}{|c|c|c|c|}
\hline $\begin{array}{l}\nu_{\max } \\
(\mathrm{Hz})\end{array}$ & $\begin{array}{c}\text { Power Spectrum } \\
(Q)\end{array}$ & $\begin{array}{l}\mathrm{rms} \\
(\%)\end{array}$ & Component \\
\hline \multicolumn{4}{|c|}{ Power Spectrum A } \\
\hline $479.73 \pm 13.65 \ldots \ldots \ldots \ldots \ldots$ & $2.47 \pm 0.52$ & $9.90 \pm 0.82$ & $L_{u}$ \\
\hline $145.80 \pm 11.15 \ldots \ldots \ldots \ldots \ldots$ & $0.58 \pm 0.17$ & $11.80 \pm 0.83$ & $L_{\mathrm{hHz}}$ \\
\hline $24.56 \pm 0.63 \ldots \ldots \ldots \ldots \ldots$ & $1.23 \pm 0.18$ & $9.80 \pm 0.67$ & $L_{h}$ \\
\hline $13.32 \pm 0.41 \ldots \ldots \ldots \ldots \ldots \ldots$ & $2.15 \pm 0.56$ & $5.39 \pm 0.84$ & $L_{\mathrm{LF}}$ \\
\hline $5.64 \pm 0.48 \ldots \ldots \ldots \ldots \ldots \ldots \ldots$ & $0.09 \pm 0.04$ & $13.77 \pm 0.47$ & $L_{b}$ \\
\hline \multicolumn{4}{|c|}{ Power Spectrum B } \\
\hline $527.99 \pm 7.32 \ldots$ & $3.25 \pm 0.41$ & $9.46 \pm 0.45$ & $L_{u}$ \\
\hline $154.20 \pm 8.80 \ldots \ldots \ldots \ldots \ldots \ldots$ & $0.72 \pm 0.14$ & $9.81 \pm 0.53$ & $L_{\mathrm{hHz}}$ \\
\hline $29.48 \pm 0.74 \ldots \ldots \ldots \ldots \ldots \ldots$ & $1.16 \pm 0.14$ & $9.15 \pm 0.52$ & $L_{h}$ \\
\hline $16.60 \pm 0.35 \ldots \ldots \ldots \ldots \ldots \ldots$ & $2.50 \pm 0.47$ & $4.91 \pm 0.60$ & $L_{\mathrm{LF}}$ \\
\hline $7.49 \pm 0.48 \ldots \ldots \ldots \ldots \ldots$ & $0.10 \pm 0.04$ & $12.94 \pm 0.35$ & $L_{b}$ \\
\hline \multicolumn{4}{|c|}{ Power Spectrum C } \\
\hline $537.98 \pm 6.68 \ldots \ldots \ldots \ldots \ldots$ & $2.98 \pm 0.34$ & $9.61 \pm 0.41$ & $L_{u}$ \\
\hline $177.08 \pm 7.90 \ldots \ldots \ldots \ldots \ldots \ldots$ & $0.82 \pm 0.14$ & $9.61 \pm 0.47$ & $L_{\mathrm{hHz}}$ \\
\hline $30.06 \pm 0.64 \ldots \ldots \ldots \ldots \ldots \ldots$ & $1.05 \pm 0.13$ & $8.83 \pm 0.49$ & $L_{h}$ \\
\hline $16.30 \pm 0.28 \ldots \ldots \ldots \ldots \ldots \ldots$ & $3.66 \pm 1.00$ & $3.39 \pm 0.52$ & $L_{\mathrm{LF}}$ \\
\hline $9.14 \pm 0.66 \ldots \ldots \ldots \ldots \ldots \ldots$ & $0.04 \pm 0.03$ & $14.05 \pm 0.40$ & $L_{b}$ \\
\hline \multicolumn{4}{|c|}{ Power Spectrum D } \\
\hline $578.98 \pm 7.30 \ldots \ldots \ldots \ldots \ldots$ & $3.71 \pm 0.47$ & $10.14 \pm 0.48$ & $L_{u}$ \\
\hline $184.59 \pm 12.70 \ldots \ldots \ldots \ldots \ldots$ & $0.86 \pm 0.23$ & $8.93 \pm 0.75$ & $L_{\mathrm{hHz}}$ \\
\hline $27.86 \pm 1.65 \ldots \ldots \ldots \ldots \ldots \ldots$ & $0.41 \pm 0.11$ & $12.73 \pm 1.23$ & $L_{h}$ \\
\hline $8.18 \pm 1.19 \ldots \ldots \ldots \ldots \ldots \ldots$ & $0.15 \pm 0.05$ & $11.06 \pm 1.18$ & $L_{b}$ \\
\hline \multicolumn{4}{|c|}{ Power Spectrum E } \\
\hline 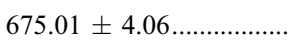 & $4.58 \pm 0.41$ & $8.54 \pm 0.27$ & $L_{u}$ \\
\hline $407.94 \pm 30.54 \ldots \ldots \ldots \ldots \ldots \ldots$ & $3.85_{-1.82}^{+5.13}$ & $2.81 \pm 0.85$ & $L_{\ell}$ \\
\hline $170.37 \pm 14.67 \ldots \ldots \ldots \ldots \ldots$ & $0.98 \pm 0.42$ & $6.65 \pm 1.44$ & $L_{\mathrm{hHz}}$ \\
\hline $61.69 \pm 3.69 \ldots \ldots \ldots \ldots \ldots \ldots \ldots$ & $0.77 \pm 0.26$ & $8.33 \pm 1.27$ & $L_{h}$ \\
\hline $29.61 \pm 0.46 \ldots \ldots \ldots \ldots \ldots \ldots$ & $2.80 \pm 0.53$ & $4.00 \pm 0.48$ & $L_{\mathrm{LF}}$ \\
\hline $14.41 \pm 0.25 \ldots \ldots \ldots \ldots \ldots \ldots$ & $1.06 \pm 0.25$ & $6.89 \pm 1.33$ & $L_{b}$ \\
\hline $9.94 \pm 3.77 \ldots \ldots \ldots \ldots \ldots \ldots \ldots$ & $0.00 \pm 0.00$ & $7.73 \pm 1.77$ & $L_{b 2}{ }^{\mathrm{a}}$ \\
\hline
\end{tabular}

Notes.-Characteristic frequencies $\nu_{\max }, O$-values $\left(\equiv \nu_{0} / \mathrm{FWHM}\right.$; see $\left.\S 2\right)$, integrated fractional rms (of the full PCA energy band), and identification (component) of the Lorentzians fitted for $4 \mathrm{U} 1820-30$. The quoted errors in $\nu_{\max }, Q$, and rms use $\Delta \chi^{2}=1.0$.

${ }^{a}$ Lorentzian with $\sim 2.7 \sigma$ significance.

To fit the power spectra, we used a multi-Lorentzian function: the sum of several Lorentzian components plus, if necessary, a power law to fit the very low frequency noise. Each of these components is usually described with an $L_{i}$ (Lorentzian) and its frequency with $\nu_{i}$, where $i$ determines the type of component. For example, $L_{u}$ identifies the upper kilohertz QPO, and $\nu_{u}$ identifies its frequency. By analogy, other components go by names such as $L_{\ell}$ (lower kilohertz), $L_{\mathrm{hHz}}$ (hectohertz), $L_{h}$ (hump), $L_{b}$ (break frequency), and their frequencies as $\nu_{\ell}, \nu_{\mathrm{hHz}}, \nu_{h}$, and $\nu_{b}$, respectively. Using this multi-Lorentzian function makes it straightforward to directly compare the different components in $4 \mathrm{U}$ $1820-30$ to those in previous works that used the same fit function (e.g., Belloni et al. 2002; van Straaten et al. [2003, 2005] and references therein).

In the fits we only include those Lorentzians with a significance larger than $3 \sigma$ based on the error in the power integrated from 0 to $\infty$. We give the results of the fits in terms of $\nu_{\max }$ and $Q$, of which $\nu_{\max }$ was introduced by Belloni et al. (2002) as $\nu_{\max }=\left[\nu_{0}^{2}+(\mathrm{FWHM} / 2)^{2}\right]^{1 / 2}=\nu_{0}\left(1+1 / 4 Q^{2}\right)^{1 / 2}$. For $Q$ we use the standard definition $Q=\nu_{0} /$ FWHM, where FWHM is the full width at half-maximum of the Lorentzian.

\section{RESULTS}

Figures 1 and 2 show that in order A-E, the spectrum becomes softer: i.e., both hard and soft color decrease, while the spectrum is harder than in the banana branch and the intensity is approximately constant (see Fig. 2). This is the expected behavior for an atoll source that is moving from the island to the lower left banana state in the color-color diagram (van der Klis 2006).

In Figure 3, we show the average power spectra with their fits. Four to five Lorentzian components were needed for a good fit of power spectra A-D. Power spectrum E, whose colors are closest to the upper banana state, could be fitted with either six or seven Lorentzians. Both fits share six components whose frequencies are the same within errors; in the case of seven Lorentzians, an extra component is present at $\nu_{3}=407.9 \pm 30.5$. This component is significant only at $\sim 2 \sigma$ (single trial) level and represents an $\sim 1.3 \sigma$ improvement of the $\chi^{2}$ of the fit according to an $F$-test. However, if this component, which is consistent with being the lower kilohertz QPO peak, is not included in the model, the fit becomes unstable unless the quality factor $Q_{\mathrm{hHz}}$ is fixed.

Tables 2 and 3 give the results of the fits to the power spectra, and in Figure 4 we show the correlations of the characteristic frequencies $\nu_{\max }$ of the power spectral components with the frequency of the upper kilohertz QPO $\nu_{u}$. For power spectrum E, we always show the results for seven Lorentzians.

As expected for the island state of an atoll source, $\nu_{u}$ is lower than $\sim 700 \mathrm{~Hz}$ (see, e.g., van Straaten et al. 2003, 2005; van der Klis 2006) and increases monotonically from A to $E$ with decreasing hard color; $L_{\mathrm{hHz}}$ is at similar frequencies as in the other atoll sources, between $\sim 100$ and $\sim 200 \mathrm{~Hz}$.

For $L_{b}$ and $L_{h}$, a shift appears to exist between the correlations of $4 \mathrm{U} 1820-30$ and those of the other atoll sources studied by van Straaten et al. (2005). To further investigate this, in Figures 5 and 6 we plot $\nu_{b}$ and $\nu_{h}$, respectively, versus $\nu_{u}$. We use all the data used by van Straaten et al. (2005) for the atoll sources and the low-luminosity bursters; however, of the millisecond pulsars, we only use data of SAX J1808.4-3658, which, in contrast to the others, has data points in the same frequency region as $4 \mathrm{U} 1820-30$. As can be seen in Figures 5 and 6, our points for $4 \mathrm{U} 1820-30$ are right in the important transition region around $\nu_{u} \sim 600 \mathrm{~Hz}$. On one hand for $L_{b}$ (Fig. 5), our points seem to link the SAX J1808.4-3658 data with those for the atoll sources with $\nu_{u} \gtrsim 600 \mathrm{~Hz}$. However, neither the frequency range covered by $4 \mathrm{U} 1820-30$ nor SAX J1808.4-3658 is sufficient to draw the conclusion that the two different correlations below $\nu_{u} \sim$ $600 \mathrm{~Hz}$ become the same correlation above $\nu_{u} \sim 600 \mathrm{~Hz}$, as the figure seems to suggest. On the other hand, as shown in Figure 6, in $4 \mathrm{U} 1820-30$ the $L_{h}$ points seem to lie between those of the atoll sources and those of SAX J1808.4-3658.

TABLE 3

Additional Results of the Fits to the Power Spectra

\begin{tabular}{|c|c|c|c|}
\hline Power Spectrum & Power-Law Index & $\begin{array}{c}\text { Power-Law } \\
\text { Parameters (rms) } \\
(\%)\end{array}$ & $\begin{array}{c}\text { Integration } \\
\text { Interval } \\
(\mathrm{Hz})\end{array}$ \\
\hline А & $1.9 \pm 0.5$ & $1.19 \pm 0.15$ & $0.01-0.08$ \\
\hline В & $1.6 \pm 0.4$ & $1.2 \pm 0.1$ & $0.01-0.06$ \\
\hline Е & $2.22 \pm 0.24$ & $1.02 \pm 0.53$ & $0.01-0.08$ \\
\hline
\end{tabular}



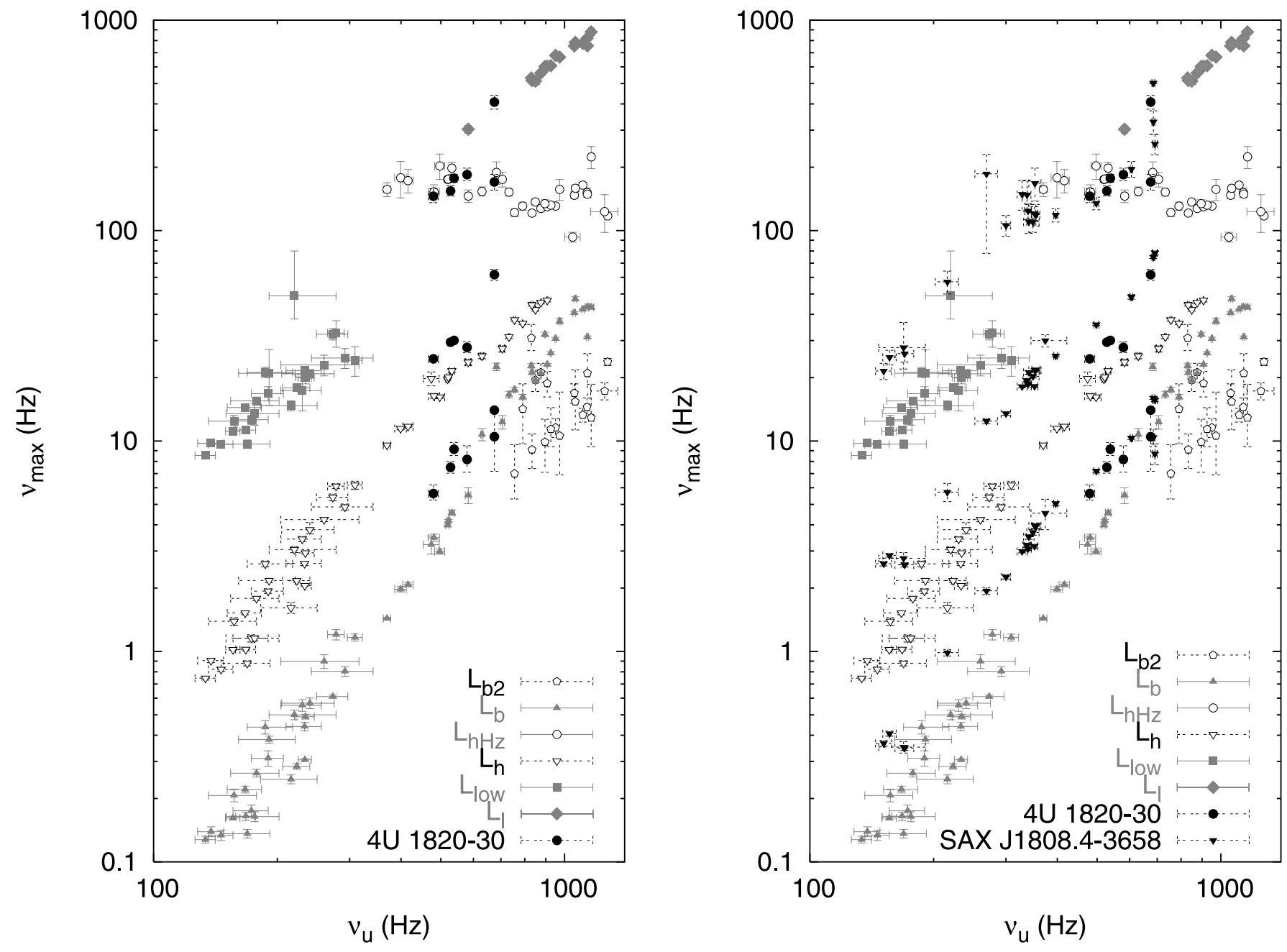

FIG. 4.-Correlations between the characteristic frequencies $\nu_{\max }$ of the various power spectral components and $\nu_{u}$. For clarity, on the left we plot the different components of the atoll sources $4 \mathrm{U} 0614+09$, 4U 1608-52, 4U 1728-34, and Aql X-1; and the low-luminosity bursters 1E 1724-3045, GS 1826-24, and SLX 1735-269 (van Straaten et al. 2005), where the filled circles mark the results for the island state features of 4U 1820-30. On the right, we show the same plot as on the left, but we include the results for the millisecond accreting pulsar SAX J1808.4-3658 (filled triangles).

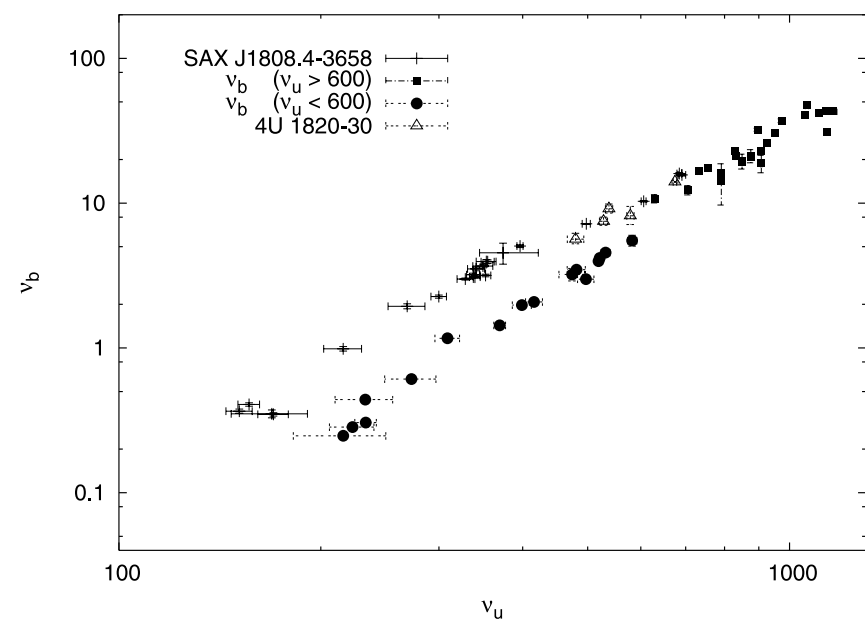

Fig. 5.-Correlation between the characteristic frequencies $\nu_{b}$ and $\nu_{u}$. The filled circles and filled squares mark the atoll sources $4 \mathrm{U} 0614+09$, $4 \mathrm{U}$ $1608-52,4 \mathrm{U} 1728-34$, and Aql X-1; and the low-luminosity bursters $1 \mathrm{E}$ 1724-3045, GS 1826-24, and SLX 1735-269 (van Straaten et al. 2005) for $\nu_{u}<600$ and $\nu_{u}>600 \mathrm{~Hz}$, respectively. The open triangles mark the results for $4 \mathrm{U} 1820-30$, and the crosses represent the results from van Straaten et al. (2005) for SAX J1808.4-3658.

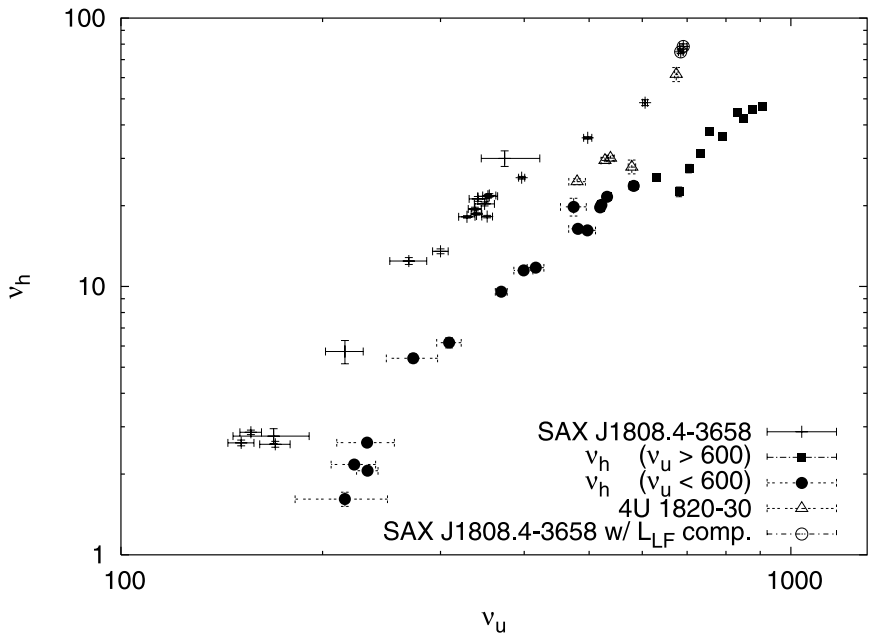

FIG. 6.-Correlation between the characteristic frequencies $\nu_{h}$ and $\nu_{u}$. Symbols are the same as in Fig. 5. The two open circles represent the results for SAX J1808.4-3658 in which a $L_{\mathrm{LF}}$ component was also found (see van Straaten et al. 2005). 
To determine the shift factors between the frequency correlations of 4U 1820-30 and those of the other atoll sources, and to be able to compare them with the shift factors found for SAX J1808.4-3658, we followed the same procedure used by van Straaten et al. (2005): we considered the $\nu_{b}$ versus $\nu_{u}$ and $\nu_{h}$ versus $\nu_{u}$ relations for which $\nu_{u}<600 \mathrm{~Hz}$, as the behavior of the low-frequency components above $600 \mathrm{~Hz}$ is complex. In practice, this means that we exclude power spectrum E. Note that in our analysis we included the data point for SAX J1808.4-3658 at $\nu_{u}=497.6 \pm 6.9 \mathrm{~Hz}$ that, when shifted, ends up above $600 \mathrm{~Hz}$ and that was excluded by van Straaten et al. (2005).

For each relation, we fitted a power law to the 4U 1820-30 frequencies together with those of the atoll sources using the FITEXY routine by Press \& Vetterling (1989), which performs a straight-line fit to data with errors in both coordinates. We took the logarithm of the frequencies so that fitting a power law becomes equivalent to fitting a straight line. Before fitting, we multiplied the $4 \mathrm{U} 1820-30 \nu_{u}$ values with a shift factor that ran between 0.1 and 3 with steps of 0.001 . The fit with the minimal $\chi^{2}$ then gave the best shift factor. The errors in the shift factor use $\Delta \chi^{2}=1$, corresponding to a $68 \%$ confidence level. The best shift factors in $\nu_{u}$ for $4 \mathrm{U} 1820-30$ are $1.21 \pm 0.02\left(\chi^{2} /\right.$ dof $\left.=19.4 / 18\right)$ and $1.13 \pm 0.01\left(\chi^{2} /\right.$ dof $\left.=45.3 / 18\right)$ for $\nu_{b}$ and $\nu_{h}$, respectively.

If we repeat the procedure described above, but this time instead of multiplying $\nu_{u}$ we multiply $\nu_{b}$ and $\nu_{h}$ by a variable factor (vertical frequency shifts in Fig. 4), the best shift factor in $\nu_{b}$ is $0.55 \pm 0.03\left(\chi^{2} / \mathrm{dof}=19.4 / 18\right)$ and in $\nu_{h}$ is $0.73 \pm 0.02$ $\left(\chi^{2} /\right.$ dof $\left.=45.3 / 18\right)$. Clearly, the high $\chi^{2}$ when calculating the best fit for $\nu_{h}$ indicates that the dispersion of the data around the power law is larger than expected from counting statistics.

In Figure 7 we plot the characteristic frequency $\nu_{h}$ versus $\nu_{b}$. As van Straaten et al. (2005) shows, the millisecond pulsar SAX J1808.4-3658 follows approximately the same correlation as the atoll sources and low-luminosity bursters at frequencies $\nu_{b} \lesssim 3 \mathrm{~Hz}$. For $3 \mathrm{~Hz} \lesssim \nu_{b} \lesssim 5 \mathrm{~Hz}$, the atoll sources slightly deviate, as $\nu_{b}$ increases, toward lower $\nu_{h}$. For $\nu_{b} \gtrsim 5$, van Straaten et al. (2005) observed a noncontinuous bifurcation where $\nu_{b}$ of the atoll sources jumps to higher frequencies while SAX J1808.4-3658 smoothly extends the correlation observed for

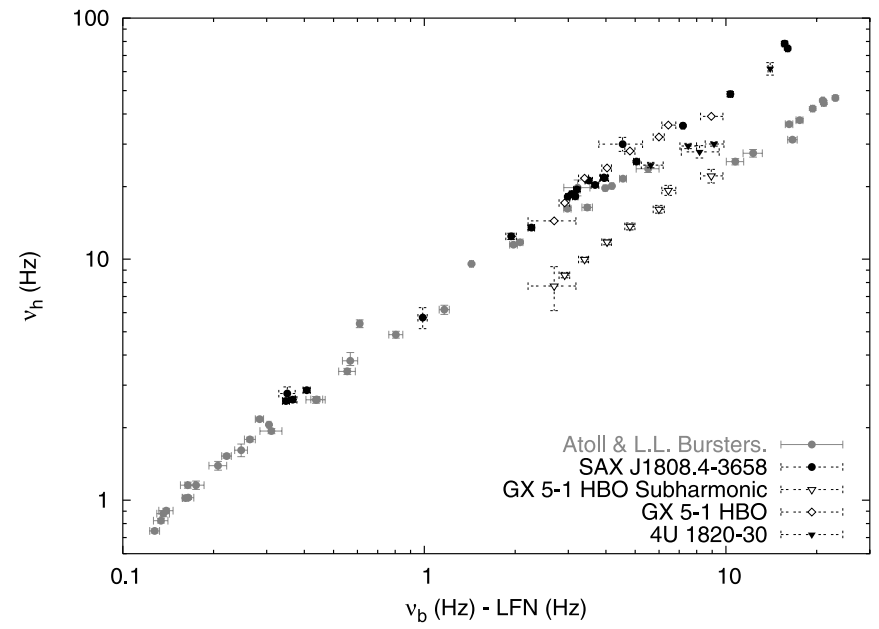

FIG. 7.-Characteristic frequency $\nu_{h}$ plotted vs. $\nu_{b}$. The gray circles mark the atoll sources 4U 0614+09, 4U 1608-52, 4U 1728-34, and Aql X-1; and the low-luminosity bursters 1E 1724-3045, GS 1826-24, and SLX 1735-269. The filled circles mark the accreting millisecond pulsar SAX J1808.4-3658 (van Straaten et al. 2005). The filled triangles mark the results for 4U 1820-30. We also include the HBO and HBO subharmonic characteristic frequencies of the Z source GX 5-1 (open diamonds and open triangles, respectively), plotted vs. that of the LFN (van Straaten et al. 2003).

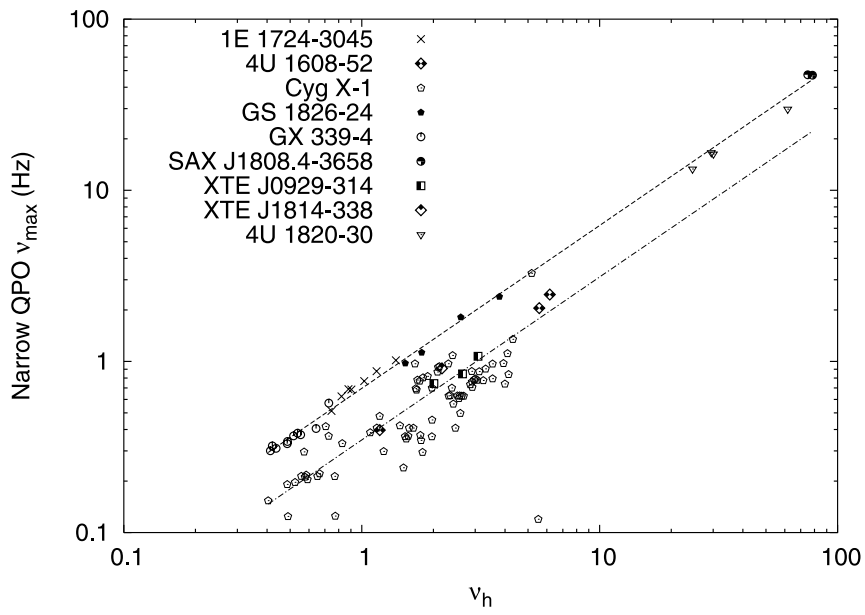

FIG. 8.-Characteristic frequencies $\nu_{\mathrm{LF}}$ and $\nu_{\mathrm{LF} / 2}$ (see text) vs. $\nu_{h}$. The symbols are labeled in the plot and represent the frequencies of the QPOs from the atoll source $4 \mathrm{U}$ 1608-52; the BHCs Cyg X-1 and GX 339-4; the lowluminosity bursters $1 \mathrm{E}$ 1724-3045 and GS 1826-24; and the accreting millisecond pulsars XTE J0929-314, XTE J1814-338, and SAX J1808.4-3658 (van Straaten et al. 2003, 2005). The open triangles show the results for $4 \mathrm{U}$ $1820-30$. The dashed line indicates a power-law fit to the $\nu_{\mathrm{LF}} \mathrm{vs}$. $\nu_{h}$ relation of the low-luminosity bursters 1E 1724-3045 and GS 1826-24, and the BHC GX $339-4$. The dash-dotted line is a power law with a normalization half of that of the dashed line. The error bars are of the order of the size of the symbols.

$\nu_{b} \lesssim 3 \mathrm{~Hz}$. Our new data for $4 \mathrm{U} 1820-30$, which are all at $\nu_{b}>5 \mathrm{~Hz}$, seem to be in between these two correlations, apparently following the behavior of the atoll sources for $3 \mathrm{~Hz} \lesssim$ $\nu_{b} \lesssim 5 \mathrm{~Hz}$. However, the point for 4U 1820-30 at higher $\nu_{b}$ (and higher $\nu_{h}$ ) falls in the correlation of SAX J1808.4-3658.

In Figure 7 we also show the frequency of the horizontal branch oscillation (HBO) and its subharmonic versus that of the low-frequency noise (LFN) for the Z source GX 5-1. The data of GX 5-1 were taken from van Straaten et al. (2003; but see Jonker et al. [2002] for original data). The HBO component of GX 5-1 follows the same correlation as SAX J1808.4-3658, but as already noted by Wijnands \& van der Klis (1999), the $\mathrm{HBO}$ of $\mathrm{Z}$ sources is slightly higher in this diagram than the $L_{h}$ and $L_{\mathrm{LF}}$ components of atoll sources. The HBO subharmonic extends the correlation that is found for atoll sources and lowluminosity bursters for $\nu_{b} \gtrsim 5 \mathrm{~Hz}$ to lower frequencies, suggesting that the apparent bifurcation mentioned before could be associated with harmonic mode switching.

In Figure 8 we plot the characteristic frequency of the narrow low-frequency QPOs $(Q \gtrsim 2.5)$, which have characteristic frequency $\nu_{\max }$ between $\nu_{b}$ and $\nu_{h}$, versus $\nu_{h}$. Such narrow QPOs were previously reported in other sources (e.g., van Straaten et al. $[2003,2005]$ and references therein), and we also detect them in 4 U 1820-30. Following van Straaten et al. (2003), for clarity we have omitted these QPOs $\left(L_{\mathrm{LF}}\right)$ from Figure 4 . In Figure 8 , the data of $4 U$ 1820-30 seem to follow the power law fitted to the $\nu_{\mathrm{LF}}$ versus $\nu_{h}$ relation of the low-luminosity bursters 1E 17243045, GS 1826-24, and the black hole candidate (BHC) GX $339-4$ by van Straaten et al. $(2003,2005)$; therefore, we identify these QPOs as being the $L_{\mathrm{LF}}$ component.

\section{DISCUSSION}

We have performed the first detailed study of the fast time variability in the island state of the atoll source 4U 1820-30. It has been reported before that the frequencies of the variability components of the atoll sources follow a universal scheme of correlations when plotted versus $\nu_{u}$ (van Straaten et al. 2003 and 
references therein). In Figure 4 (left panel) we show that our data are in general agreement with this scheme; van Straaten et al. (2005) showed that the accreting millisecond pulsar SAX J1808.4-3658 shows similar relations between its characteristic frequencies as the atoll sources do, but shifted (Fig. 4, right panel). This shift was interpreted to occur only between the characteristic frequencies of the low-frequency components on one hand and $\nu_{u}$ (and $\nu_{\ell}$ ) on the other, where $\nu_{u}$ (and $\nu_{\ell}$ ) had to be multiplied by $\sim 1.45$ to make the correlations coincide. Figures 5 and 6 suggest that this could also be the case for $4 \mathrm{U}$ $1820-30$. However, the shift factor for $\nu_{u}$ is $1.21 \pm 0.02$ and $1.13 \pm 0.01$ for $L_{b}$ and $L_{h}$, respectively, giving an average of $1.17 \pm 0.01$, which is smaller than the values of $1.420 \pm 0.013$ and $1.481 \pm 0.013$, respectively, giving an average of $1.454 \pm$ 0.009 (van Straaten et al. 2005). Similar shift factors as we find for $4 \mathrm{U}$ 1820-30 may in fact be present in other accreting millisecond pulsars and faint burst sources; for example, in XTE J1751-305 van Straaten et al. (2005) found shift factors of $1.188 \pm 0.045$ and $1.112 \pm 0.042$ for $L_{b}$ and $L_{h}$, respectively. These results are consistent with our values; however, the results for XTE J1751-305 have larger errors. It is important to note that XTE J1751-305 has a companion of $0.013-0.35 M_{\odot}$, suggesting a heated helium dwarf (Markwardt et al. 2002). Since $4 \mathrm{U} 1820-30$ also has a low-mass helium dwarf, the similarity in frequency shifts might be related to the chemical composition of the material in the accreting disk. However, a simple "frequency shift-chemical composition" relation is not evident, since the composition of the companion stars of SAX J1808.4-3658, 4U $0614+09,4 \mathrm{U} 1608-52$, and $4 \mathrm{U} 1728-34$ are uncertain. For instance, SAX J1808.4-3658 might have a brown dwarf, 4U 0614+09 might have an oxygen-carbon white dwarf, and both $4 \mathrm{U} 1608-52$ and $4 \mathrm{U}$ 1728-34 might have late-type mainsequence companions (but see Bildsten \& Chakrabarty [2001], Nelemans et al. [2004], Wachter et al. [2002], and Marti et al. [1998], respectively, for discussions).

Authors van Straaten et al. (2005) suggested that the measured shift factors of $\sim 1.5=\sim 3 / 2$ could be related with the parametric resonance models for kilohertz QPOs (e.g., Abramowicz et al. 2003), where the $2: 3$ frequency resonances between general relativistic orbital/epicyclic frequencies play a central role. The average shift factor for $4 \mathrm{U} 1820-30$ is $1.17 \pm 0.01$, so we can reject the idea that $2: 3$ resonances are the (only) cause of the shifts.

We further attempted to test the hypothesis that a multiplicative shift of frequencies is the right interpretation of the difference in the frequency correlations between SAX J1808.4-3658 and the other atoll sources. If that hypothesis is correct, we should expect both correlations to have the same power-law index within errors, and the only significant difference between the correlations would arise from the normalization of each of the power laws. In order to quantify the differences, we performed two different fits in which simultaneously a power law is fitted to the data of SAX J1808.4-3658 and another power law is fitted to the data of the atoll sources $4 \mathrm{U} 1608-52,4 \mathrm{U} 0614+09$, and $4 \mathrm{U} 1728-34$. Then we compare the $\chi^{2} /$ degrees of freedom (dof) of the fits. We only use data of the $L_{b}$ components, since $L_{h}$ 's behavior is more complex. If both power-law indexes and normalizations are free parameters, the best fit gives a $\chi^{2} / \mathrm{dof}=60.2 / 30$. If we force both power laws to have the same index, but different normalizations, the best fit gives a $\chi^{2} /$ dof $=86.2 / 31$.

By comparing these results using an $F$-test, we find that the improvement of the fit when leaving all the parameters free as compared to forcing equal slopes is significant at the $3.4 \sigma$ level. If the "shift" interpretation is correct, then the slopes of both correlations should be the same, and we should not find a significant improvement of the fit. However, the fact that we are dealing with $\chi^{2} /$ dof $\gtrsim 2$ reduces the statistical significance of our possible interpretations.

If we perform the same analysis between $4 \mathrm{U} 1820-30$ and the atoll sources, we find $\chi_{1}^{2} /$ dof values of $19.4 / 16$ and 19.6/17, i.e., no significant improvement of the fit. Therefore, in both cases the data are not inconsistent with the hypothesis that the differences between correlations are due to only a shift in $\nu_{u}$ (van Straaten et al. 2005).

As suggested by van Straaten et al. (2005), the simplest explanation for the shift between correlations is that there is some physical difference between sources that affects $\nu_{u}$. Up to now, such shifts had only been seen in accreting pulsars and only at high confidence in SAX J1808.4-3658, which led to the suggestion that the same source property that leads to strong pulsations also affects $\nu_{u}$ (van Straaten et al. 2005). 4U 182030 has no strong pulsations (Dib et al. 2005), invalidating any strict relation between these two characteristics. However, as the shifts in $4 \mathrm{U} 1820-30$ are smaller than in SAX J1808.43658 , and as accidental circumstances such as an unfavorable viewing geometry could suppress the pulsations in $4 \mathrm{U} 1820-$ 30 , it is too early to reject this idea.

D. A. would like to thank M. Linares and R. Wijnands for helpful discussions. This work was supported by the Nederlandse Onderzoekschool Voor Astronomie (NOVA), i.e., the Netherlands Research School for Astronomy, and it has made use of data obtained through the High Energy Astrophysics Science Archive Research Center (HEASARC) Online Service, provided by the NASA Goddard Space Flight Center.

\section{REFERENCES}

Abramowicz, M. A., Karas, V., Kluzniak, W., Lee, W. H., \& Rebusco, P. 2003, PASJ, 55, 467

Belloni, T., Psaltis, D., \& van der Klis, M. 2002, ApJ, 572, 392

Bildsten, L., \& Chakrabarty, D. 2001, ApJ, 557, 292

Bloser, P. F., Grindlay, J. E., Kaaret, P., Zhang, W., Smale, A. P., \& Barret, D. 2000, ApJ, 542, 1000

Dib, R., Ransom, S., Ray, P., Kaspi, V., \& Archibald, A. 2005, ApJ, 626, 333 Geldzahler, B. J. 1983, ApJ, 264, L49

Grindlay, J., Gursky, H., Schnopper, H., Parsignault, D. R., Heise, J., Brinkman, A. C., \& Schrijver, J. 1976, ApJ, 205, L127

Hasinger, G., \& van der Klis, M. 1989, A\&A, 225, 79

Jahoda, K., Swank, J. H., Giles, A. B., Stark, M. J., Strohmayer, T., Zhang, W., \& Morgan, E. H. 1996, Proc. SPIE, 2808, 59

Jonker, P. G., van der Klis, M., Homan, J., Méndez, M., Lewin, W. H. G., Wijnands, R., \& Zhang, W. 2002, MNRAS, 333, 665
Kaaret, P., Piraino, S., Bloser, P. F., Ford, E. C., Grindlay, J. E., Santangelo, A., Smale, A. P., \& Zhang, W. 1999, ApJ, 520, L37

Kuulkers, E., van der Klis, M., Oosterbroek, T., Asai, K., Dotani, T., van Paradijs, J., \& Lewin, W. H. G. 1994, A\&A, 289, 795 2003, A\&A, 399, 663

Markwardt, C. B., Swank, J. H., Strohmayer, T. E., in’t Zand, J. J. M., \& Marshall, F. E. 2002, ApJ, 575, L21

Marti, J., Mirabel, I. F., Rodriguez, L. F., \& Chaty, S. 1998, A\&A, 332, L45

Méndez, M. 2002, in Proc. 9th Marcel Grossman Meeting, ed. V. Gurzadyan, R. Jantzen, \& R. Ruffini (Signapore: World Scientific), 2319

Migliari, S., Fender, R. P., Rupen, M., Wachter, S., Jonker, P. G., Homan, J., \& van der Klis, M. 2004, MNRAS, 351, 186

Miller, M. C., Lamb, F. K., \& Psaltis, D. 1998, ApJ, 508, 791

Nelemans, G., Jonker, P. G., Marsh, T. R., \& van der Klis, M. 2004, MNRAS, 348, L7 
Press, W. H., Flannery, B. P., \& Vetterling, W. T. 1989, Numerical Recipes in Fortran (Cambridge: Cambridge Univ. Press)

Priedhorsky, W., \& Terrell, J. 1984, ApJ, 284, L17

Rappaport, S., Ma, C. P., Joss, P. C., \& Nelson, L. A. 1987, ApJ, 322, 842

Revnivtsev, M., Churazov, E., Gilfanov, M., \& Sunyaev, R. 2001, A\&A, 372, 138

Smale, A. P., Zhang, W., \& White, N. E. 1997, ApJ, 483, L119

Stella, L., Priedhorsky, W., \& White, N. E. 1987, ApJ, 312, L17

Strohmayer, T., \& Bildsten, L. 2006, in Compact Stellar X-Ray Sources, ed

W. H. G. Lewin \& M. van der Klis (Cambridge: Cambridge Univ. Press), in press

Strohmayer, T. E., \& Brown, E. F. 2002, ApJ, 566, 1045

van der Klis, M. 1995, in The Lives of the Neutron Stars, ed. M. A. Alpar, U. Kiziloglu, \& J. van Paradijs (Dordrecht: Kluwer), 301 2000, ARA\&A, 38, 717 van der Klis, M. 2001, ApJ, 561, 943

2006, in Compact Stellar X-Ray Sources, ed. W. H. G. Lewin \& M. van der Klis (Cambridge: Cambridge Univ. Press), in press

van Straaten, S., van der Klis, M., \& Méndez, M. 2003, ApJ, 596, 1155

van Straaten, S., van der Klis, M., \& Wijnands, R. 2005, ApJ, 619, 455

Wachter, S., Hoard, D. W., Bailyn, C. D., Corbel, S., \& Kaaret, P. 2002, ApJ, 568,901

Wijnands, R., \& van der Klis, M. 1999, ApJ, 514, 939

Zhang, W., Giles, A. B., Jahoda, K., Soong, Y., Swank, J. H., \& Morgan, E. H. 1993, Proc. SPIE, 2006, 324

Zhang, W., Jahoda, K., Swank, J. H., Morgan, E. H., \& Giles, A. B. 1995, ApJ, 449, 930

Zhang, W., Smale, A. P., Strohmayer, T. E., \& Swank, J. H. 1998, ApJ, 500, L171 